\title{
Fatal encephalopathy complicating lymphoid interstitial pneumonia
}

\author{
MICHAEL JEFFERSON, D. RIDDOCH, ${ }^{1}$ AND W. THOMAS SMITH \\ From the Department of Neurology, United Birmingham Hospitals; Depurtment of Pathology, \\ University of Birmingham
}

SUMMARY The case is reported of a young woman who suffered from lymphoid interstitial pneumonia. Involvement of the brain by what appeared to be an identical pathological process led to her death, and the orbital tissues were also involved at one stage. The cause of this condition is unknown, but the hypotheses are put forward that it may represent one facet of delayed hypersensitivity or that a slow virus is the responsible aetiological agent. Although lymphoid interstitial pneumonia has previously been reported as an isolated entity, the evidence from this case suggests that it might represent part of a pluri-systemic disease.

Lymphoid interstitial pneumonia (LIP) was described by Liebow (1968) as a distinct variety of interstitial pneumonia. He noted that in LIP the histological appearances in the lung may resemble those of Hashimoto's thyroiditis and also that anti-lung antibodies may be elevated; he suggested, therefore, that the process might be an autoimmune phenomenon. This report describes a patient suffering from LIP who subsequently developed a relapsingremitting and eventually fatal neurological illness that resulted from an encephalitic process characterized by a cellular infiltrate in the brain resembling that in the lung.

\section{CASE REPORT}

A 22 year old married woman was first admitted to hospital in February 1965. One week previously, for the first time in her life, she had had a major fit in which her head and eyes turned to the left. This was followed in the next few days by five brief attacks of focal left-sided sensory epilepsy, each starting with tingling in the hand spreading to involve the face, trunk and leg.

As a child her tonsils, adenoids, and appendix had been removed. At the age of 13 , she was taken to a chest clinic with a history of persistent cough for one month. Chest radiographs showed an opacity at the left base and some pleural reaction; the Heaf test was positive. Treatment with penicillin resulted in complete

Applications for reprints should be addressed to D.R. clinical and radiological resolution in six weeks. For some years she had been subject to mild eczema.

Clinical examination on admission was normal. Investigations showed: $\mathrm{Hb} 12.7 \mathrm{~g} / 100 \mathrm{ml}$; WBC 4,200/ c.mm, with a normal differential count; ESR $2 \mathrm{~mm} / \mathrm{hr}$ (Westergren); blood Wassermann reaction negative. The CSF was clear and under normal pressure, with $5 \mathrm{WBC} / \mathrm{c} . \mathrm{mm}$, protein $40 \mathrm{mg} / 100 \mathrm{ml}$, Lange curve normal, and WR negative; EEG, radiographs of the chest and skull, and a right carotid angiogram were all normal.

She was discharged from hospital on phenobarbitone $30 \mathrm{mg}$ b.d. Over the following year she remained well and free of fits, until she developed uncomplicated herpes zoster of the right chest, which was followed by an isolated epileptic attack; the dose of phenobarbitone was increased to thrice daily. She later became pregnant, and during labour deep transverse arrest of the foetus required forceps delivery. Afterward she began taking an oral contraceptive (Lyndiol).

In May 1967, over a period of a week, she developed swelling and closure of the right eyelids, with milder changes on the left. When admitted to hospital, three weeks later, her signs had partially resolved, but she still showed bilateral ptosis, more marked on the right, deficient elevation of the right eye, and a slightly dilated right pupil. There was also oedema of both eyelids, of the conjunctivae, and of the forehead. There was postherpetic scarring of the right chest wall, and a mild eczematous rash on the neck and elbows. The spleen was palpable but there was no hepatomegaly or significant lymphadenopathy. The heart and lungs were normal and the blood pressure $130 / 70 \mathrm{~mm} \mathrm{Hg}$.

Laboratory investigations now revealed the following: 
Hb $14.0 \mathrm{~g} / 100 \mathrm{ml}$.; WBC $5,600 / \mathrm{c} . \mathrm{mm}$ with a normal differential count; platelets $145,000 /$ c.mm; ESR $9 \mathrm{~mm} / \mathrm{hr}$. Serum urea, electrolytes, cholesterol, alkaline phosphatase, glutamic-oxaloacetic transaminase, uric acid, and protein-bound-iodine were all normal; serum albumin $4 \cdot 2 \mathrm{~g}$ and globulin $2 \cdot 2 \mathrm{~g} / 100 \mathrm{ml}$. with a normal electrophoretic pattern; LE cells were not seen in two specimens of blood. The Paul-Bunnell, Rose-Waaler, and latex fixation tests were negative, as were tests for fluorescent anti-nuclear factor, and complement fixations for viruses and autoimmune tissue antibodies. The CSF pressure, chemistry, and cytology were normal. The EEG and electrocardiogram were normal. Vertebral angiography was unremarkable. However, radiographs of the chest now revealed multiple soft opacities at both lung bases, which persisted in serial films. Sputum examination showed no evidence of tuberculosis or other infection. Lung function was normal, The Mantoux test was negative at 1 in 1,000 .

The ocular symptoms and signs gradually subsided, but in view of the persistently abnormal chest radiographs, lung biopsy was carried out. At thoracotomy, both upper and lower lobes were studded with small dark nodules. Histologically the biopsy showed that these nodular foci (Fig. 1) consisted of areas in which there was alveolar collapse and extensive lymphocytic infiltration, particularly around bronchioles and small arteries (Fig. 2), and in the interlobular septa. Germinal centres were present and variable numbers of plasma cells and foamy macrophages were admixed with the lymphocytic infiltrate. There was mild fibrosis within the nodules but the intervening lung looked normal. No organisms or birefringent particles were seen. It was concluded that the process was probably inflammatory and not a reticulosis.

By the time the patient was discharged from hospital her ptosis, ocular paralysis, and the local oedema had allo virtually gone, but the diagnosis was still obscure. It seemed that she might have some unusual inflammatory affection, conceivably of the delayed hypersensitivity ${ }_{0}$ variety (type IV, Gell and Coombs, 1968), involving the orbital tissues as well as the lungs. Treatment with prednisone $30 \mathrm{mg}$ daily was initiated, the dose being soon reduced to $5 \mathrm{mg}$ daily, which was continued for the. next 12 months. During this time she remained well (except for three brief attacks of left-sided paraesthesia) though serial radiographs of the chest showed little들 change.

In June 1968 she again had a major fit, preceded by three attacks of focal twitching, this time right sided. $\mathbb{\nabla}$ After a few days she was noticed to be a little confused and she complained of intense headaches. On admission's to hospital a fortnight later she looked unwell but was $\vec{\circ}$ afebrile. Neurological examination revealed dysphasia, right homonymous hemianopia, mild right hemiparesis, $\vec{\omega}$ and early papilloedema on the right. The chest was clear? clinically. There was moderate hepatosplenomegaly but? no lymphadenopathy. Investigation showed: $\mathrm{Hb} 14.00$ g/100 ml.; normal WBC; ESR $7 \mathrm{~mm} / \mathrm{hr}$; no LE cells; urinalysis negative; normal serum urea, electrolyt calcium, phosphorus, sugar, proteins, and liver functienci tests. Serum protein immunophoresis revealed low levgis $\rightarrow$ of $\operatorname{IgG}(340 \mathrm{mg} / 100 \mathrm{ml}$.$) and \operatorname{IgA}(18 \mathrm{mg} / 100 \mathrm{ml}$.), boito normal IgM $(80 \mathrm{mg} / 100 \mathrm{ml}$.) and no detectable IgS. Bleeding, clotting, and prothrombin times were normal, Coombs's test was negative and there was a norn?a:

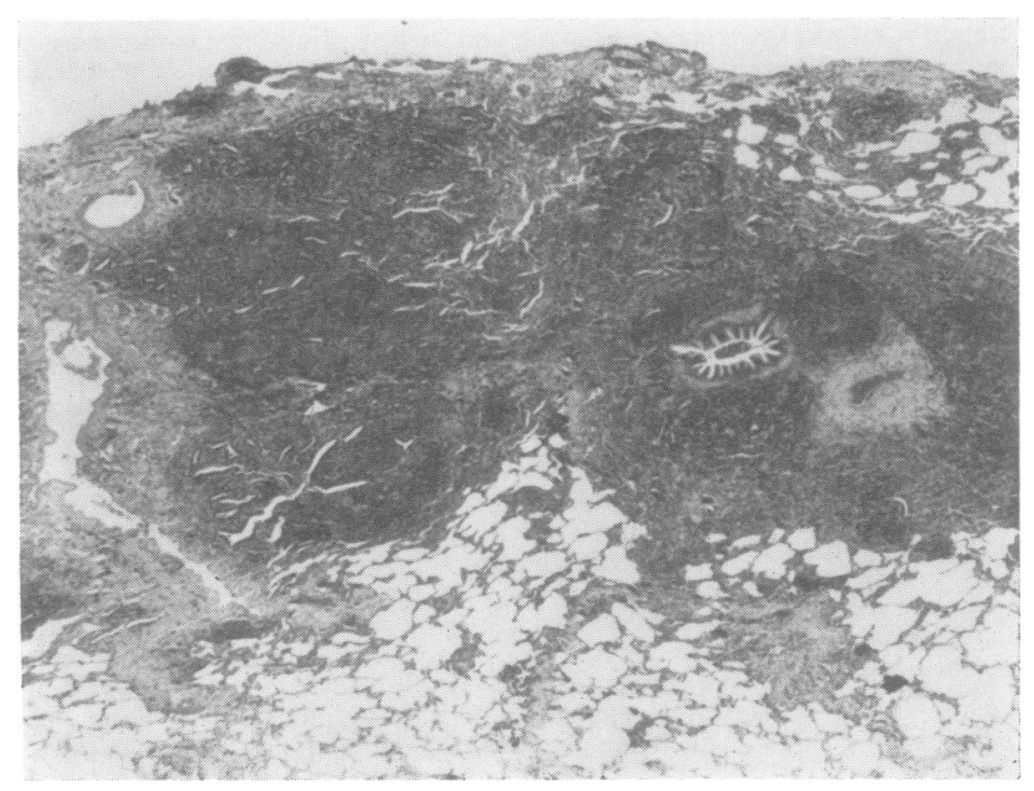

FIG. 1. Lung biopsy showing lymphocytic infiltration. $H$ and $E, \times 18$. 


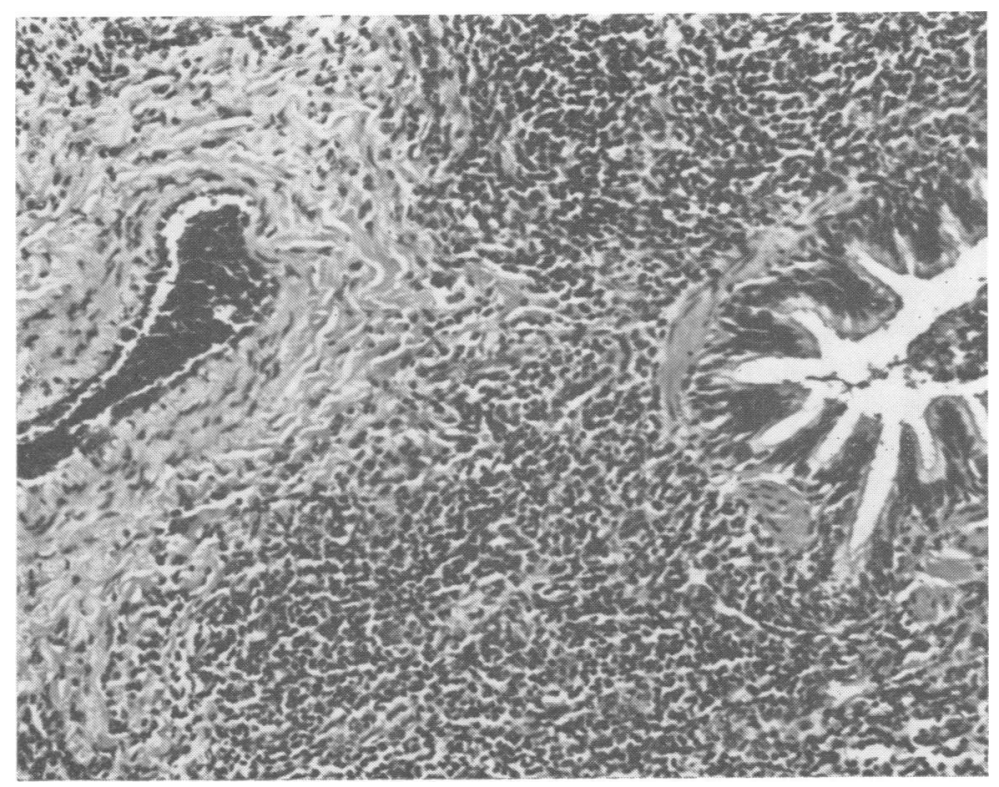

FIG. 2. Enlarged view of

Fig. 1, showing peribronchiolar and perivascular lymphocytic infiltration. $H$ and $E, \times 120$. haptoglobin level; serum vitamin $\mathbf{B}_{12}$ level was $700 \mathrm{pg} / \mathrm{ml}$.; serum iron $18 \mathrm{mg} / 100 \mathrm{ml}$. Toxoplasma dye test was negative; viral and tissue complement-fixation tests were negative. Chest radiographs showed essentially the same appearance as before.

Needle biopsy of the liver was taken which only showed mild lymphocytic cuffing of the portal tracts; there was no evidence of reticulosis, sarcoidosis or other lesions.
EEG revealed a delta wave focus centred over the left temporal lobe, and carotid angiography confirmed an avascular space occupying lesion in this situation. When exploratory craniotomy was performed, the temporal lobe was seen to be swollen and soft, the macroscopic appearance suggesting a malignant glioma. Biopsy was taken and the skull closed. Histologically there was a diffuse encephalitic process (Fig. 3) with extensive demyelination, and proliferation of large atypical

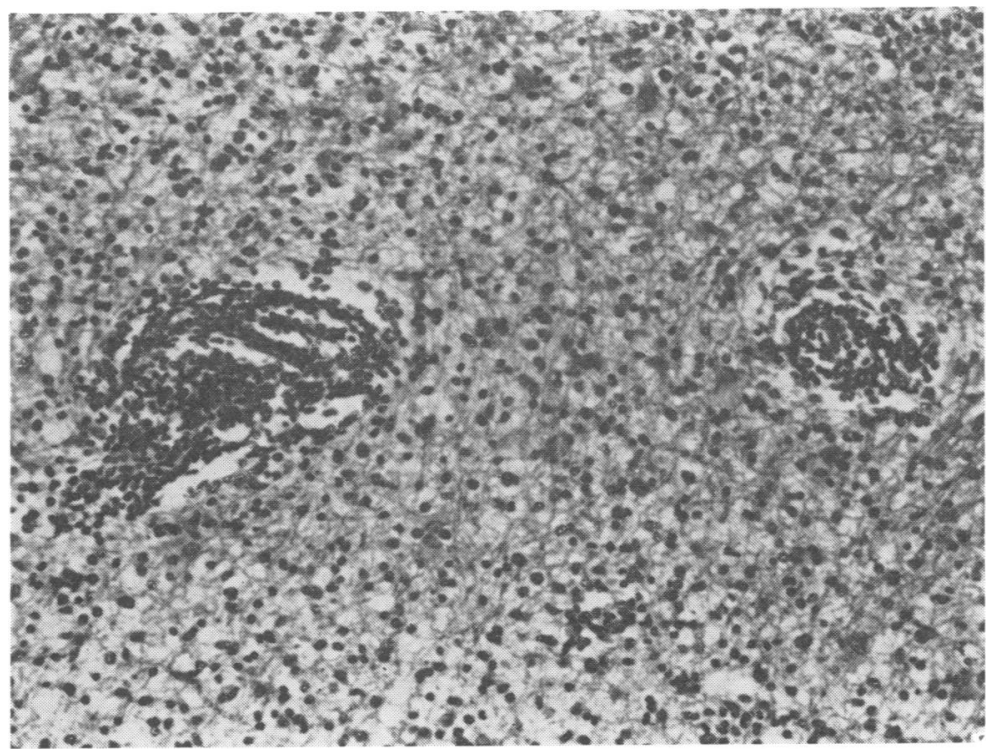

FIG. 3. Cerebral biopsy showing perivascular and more diffuse infiltration of the white matter with chronic inflammatory cells. There is diffuse demyelination. Luxol fast blue-cresyl fast violet $\times 120$. 
multinucleated and feebly fibre-forming gemistocytic astrocytes (Fig. 4). There was a pronounced infiltration with lymphocytes and plasma cells, forming broad cuffs around small blood vessels and spreading out beyond the Virchow-Robin spaces into the cerebral substance. There were many large binucleate intensely pyrinophilic plasma cells (? plasmablasts). The tissue disorganization was so severe that it was not possible to differentiate between grey and white matter. Organisms were not identified. A few oligodendrocytes contained eosinophilic intranuclear inclusions and the question of progressive multifocal leucoencephalopathy was raised. With this in mind the previous lung biopsy was re-assessed but it was again concluded that the lesions present could not be regarded as those of a reticulosis. An inflammatory process (? allergic) affecting the brain was the final conclusion.

After craniotomy her condition steadily improved. Three weeks later the only neurological signs were a mild expressive dysphasia, and weakness of the left lateral rectus muscle which had appeared after admission and was now subsiding. She was allowed home on prednisone $2.5 \mathrm{mg}$ daily along with phenobarbitone.

A week later she had to be readmitted on account of violent headache and vomiting which had developed three days previously. At this time the only abnormal neurological sign was the residual weakness of the left lateral rectus muscle, but two days later acute bilateral papilloedema was seen and on the same day she collapsed with cardiorespiratory arrest. Despite intubation and artificial pulmonary ventilation, she remained unconscious and hypotensive with fixed dilated pupils. An echoencephalogram showed no shift of midline structures, and attempts to needle the lateral ventricles were unsuccessful. Bilateral subtemporal decompression was performed, and the brain was found to be very swollen.
Biopsy material was taken from both temporal regionsy That from the left side showed microscopic feature $\$$ identical with those previously seen; the cortex was severely affected but there were no intraneuronal ince clusions. Tissue from the right temporal biopsy was much less affected. There was also evidence of mild meningeat infiltration. Terminal cardiac arrest occurred two dax after the operation.

NECROPSY FINDINGS The body $(173 \mathrm{~cm})$ was thi $(41 \mathrm{~kg})$ and showed no relevant external abnormalities.

The teeth, tongue, mouth cavity, cervical lymph nodes salivary glands, and thyroid were all unremarkable. The innominate, carotid, subclavian, and vertebral arteries appeared to be free from disease.

Respiratory tract Pleural cavities were normal. The peribronchial, subcarinal, and paratracheal lymph nodes were all moderately enlarged, of soft fleshy consistency and discrete. The trachea and proximal bronchi showed mucosal congestion and oedema and contained muco purulent exudate. There were numerous nodules, varying from a few $\mathrm{mm}$ to about 2 to $3 \mathrm{~cm}$ in diameter, scattere throughout both lungs, especially in the basal zones The nodules were firm, bulged above the pleural and cut surfaces, were paler than the surrounding lung, and did not exude pus. The smaller nodules were mainly algng the lung margins. There was no diffuse fibrosis, "hon comb', or cystic degeneration or emphysema.

The heart $(250 \mathrm{~g})$ showed no right-sided hypertropis or other relevant changes.

The thymus was replaced by enlarged lymph nodes

Abdominal lymph nodes The mesenteric, para-aotic and iliac groups, were enlarged but of normal cônं sistency, resembling the thoracic nodes.

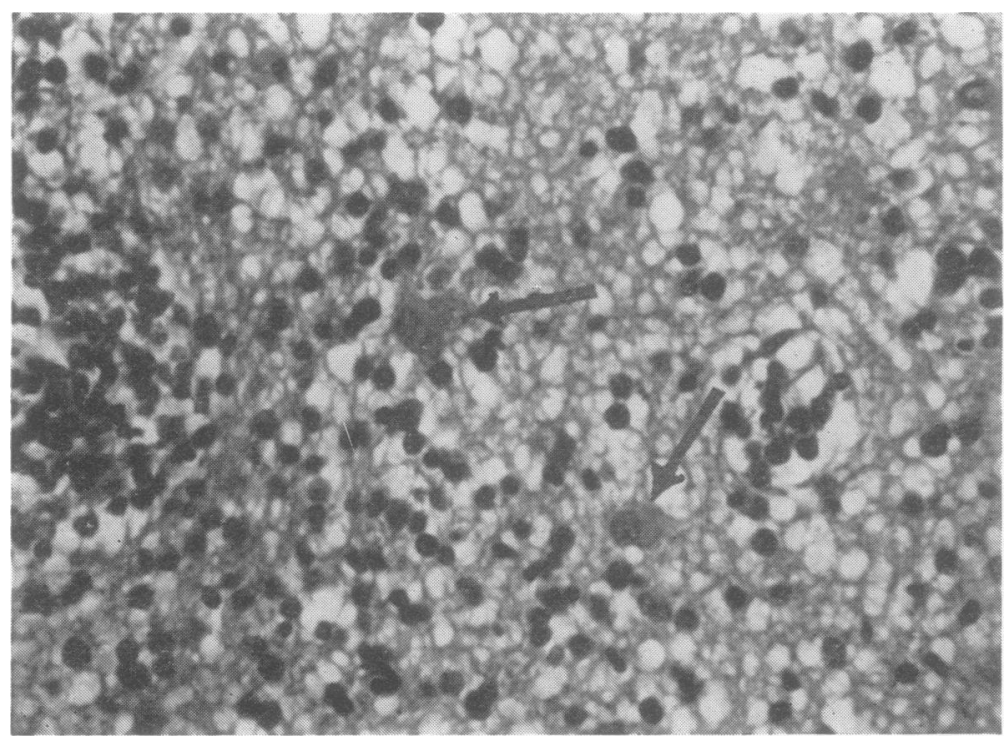

FIG. 4. Demyelinated white matter showing nodular and diffuse infiltration with lymphocytes and plasma cells.:Note large stellate astrocytes 3 (arrows). Luxol fast bluecresyl fast violet $\times 300$. 
The spleen $(350 \mathrm{~g})$ was enlarged $(23 \times 13 \times 5 \mathrm{~cm})$ showing a soft, mushy pulp but no other changes.

The liver $(2,100 \mathrm{~g})$ was enlarged, pale yellow in colour, and had a slightly greasy cut surface. There were no focal lesions.

The gall bladder was normal. The bile-ducts were patent.

The kidneys $(280 \mathrm{~g})$, ureters, stomach, intestines, pancreas, adrenals, peritoneal cavity, bladder, uterus, Fallopian tubes, ovaries, abdominal aorta and inferior vena cava, skeletal muscles, and peripheral nerves were all unremarkable.

Brain The dura mater was extremely tense. The venous sinuses were patent. There was gross symmetrical swelling of both cerebral hemispheres with flattening of the convolutions and obliteration of sulci. Both temporal lobes bulged for about $2 \mathrm{~cm}$ through bilateral temporalcraniotomy decompression sites. The cerebral hemispheres were extremely soft and friable and showed pink-blue ('bruised') discolouration due to 'respirator autolysis'; as a result, the cerebrum was difficult to remove and tended to disintegrate on handling. The cerebellum was similar but the brain-stem was firmer. The floor of the third ventricle was tense and bulged over a flattened pituitary gland.

HISTOLOGY Lungs These showed numerous, interstitial nodules similar to those seen in the biopsy material but less florid. Mild suppurative bronchopneumonia was superimposed.

Heart The myocardium showed intermuscular oedema and mild to moderate diffuse infiltration with lymphocytes and plasma cells (Fig. 5). The muscle fibres showed fatty degeneration. The appearances indicated a nonspecific myocarditis.

Liver The portal tracts showed mild infiltration with lymphocytes and plasma cells (Fig. 6), and the liver cells showed fatty vacuolation. There was fine portal fibrosis but no necrosis of liver cells.

Adrenals These showed cortical atrophy (on steroids).

Spleen and lymph nodes They showed non-specific reactive hyperplasia with follicular enlargement and sinus catarrh. The tissue architecture was retained and there was no evidence of reticulosis.

The uterus, pancreas, pituitary, tongue, skeletal muscles, and peripheral nerves were all unremarkable.

Brain Widespread autolytic changes made interpretation difficult. A diffuse encephalitis was nevertheless still recognizable and particularly prominent in the temporal and parietal areas. The subependymal regions and meninges also showed heavy infiltration with inflammatory cells. The character of the inflammation was identical with that seen in the biopsies. Both grey and white matter were involved.

\section{DISCUSSION}

Throughout three and a half years during which this young woman was under observation, the nature of her relapsing and remitting illness remained a mystery. There was doubt, never finally resolved, about its exact duration, as it was not clear whether the pulmonary affection at the age of 13 might have

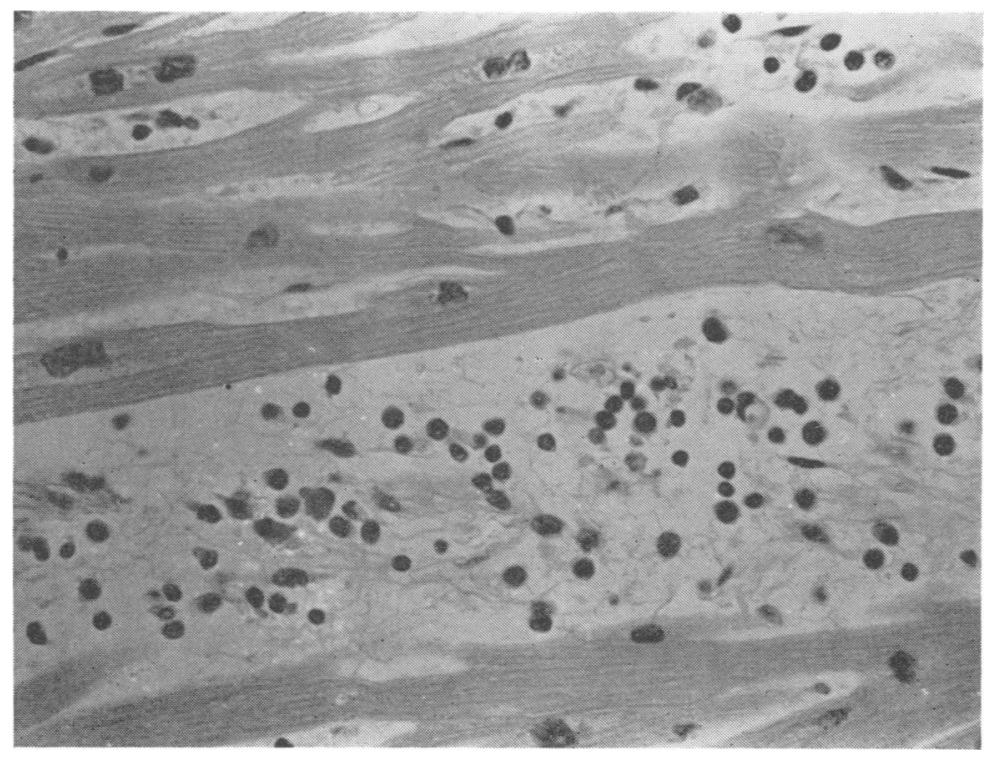

FIG. 5. Myocardium showing intermuscular oedema and infiltration with lymphocytes and plasma cells. $H$ and $E$, $\times 300$. 


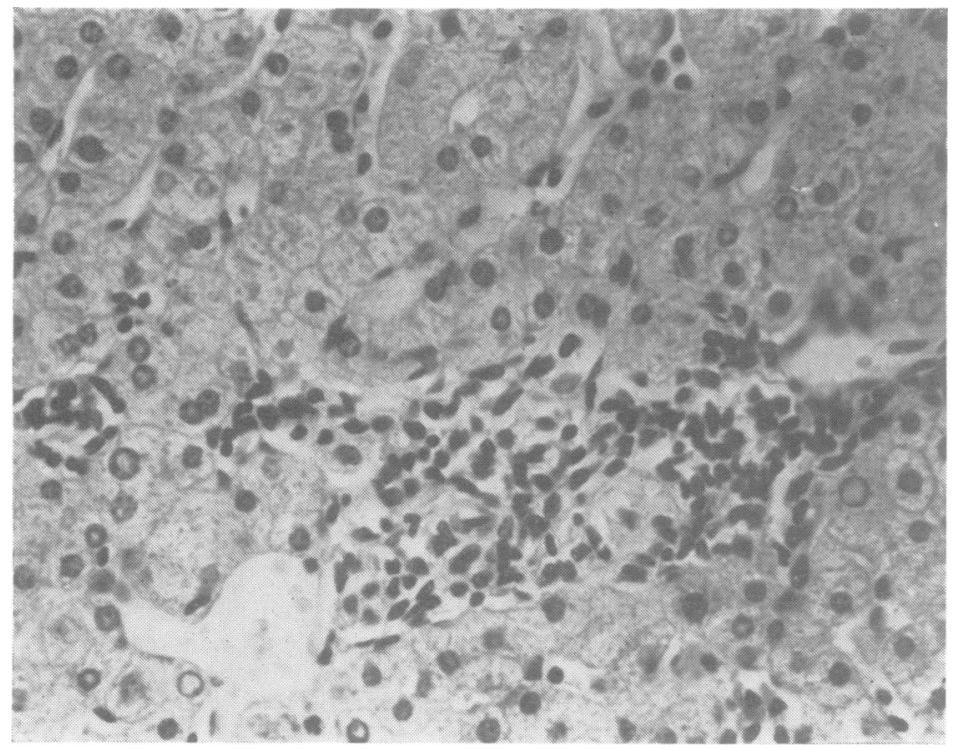

FIG. 6. Liver showing focal infiltration with chronic inflammatory cells. $\mathrm{H}$ and $\mathrm{E}$, $\times 300$

marked the opening phase of her illness, or whether this incident was aetiologically unrelated. It was thought most likely that she was suffering from some form of allergic inflammatory disorder, possibly Wegener's granulomatosis or sarcoidosis, and on this assumption she was given steroid therapy but without detectable benefit. Later the diagnosis of reticulosis with predominantly cerebral manifestations and superficial lymph node escape was considered to be an alternative possibility. The final diagnosis of lymphoid interstitial pneumonia (LIP) with widespread dissemination of lesions outside the lung was only reached after post-mortem study.

Histologically, the lung lesions in this case are unequivocally those of LIP as described by Liebow (1968). The presence of lymphoid follicles and plasma cells, many of the latter being atypical, suggests a microscopical resemblance to autoimmune disorders such as Hashimoto's thyroiditis and Sjögren's syndrome. There is also some histological affinity to the pulmonary lesions of farmer's lung (Seal, Hapke, Thomas, Meek, and Hayes, 1968), though in the latter condition the pathological changes are not usually focal, and are accompanied by cystic and emphysematous areas as well as by vascular lesions from pulmonary hypertension and cor pulmonale. But there is no evidence that the young woman had been exposed to risk from inhaled antigenic material, to cause a hypersensitivity reaction as in farmer's lung.

With regard to the histological picture found in the brain at necropsy, although there were widespread autolytic changes due to 'respirator death', this did not conceal a diffuse encephalitic process $\stackrel{+}{\omega}$ most marked temporoparietally and identical wi that seen originally in the cerebral biopsy materian; $\vec{A}$ there was also meningeal and ependymal involvis-o ment.

There was some histological similarity to progressive multifocal leucoencephalopathy, notably 贯穴 the presence of large bizarre astrocytes, oligo: $\vec{\varphi}$ dendrocytic inclusions, and demyelination. The clinical evolution of the illness was atypical of that affection, and there were no signs of reticulosis, blood dyscrasia, or the other disorders that have most often been linked with it. Organisms suggestive $\bar{O}$ of an infective or parasitic process were not seen in $\frac{\mathscr{D}}{\not{Q}}$ the cerebral tissue.

A slow or latent virus infection merits con- $\overrightarrow{\overrightarrow{0}}$ sideration. There are two points of special interest 3 in this connexion, which might predicate a viral cause, arising against a background of diminished? host resistance. Firstly, the $A$ and $G$ immunoglobulin factors of her serum were both distinctlyz low, a feature that may reflect an underlying weak- - ness of bodily defence mechanisms. Secondly, during her illness she had an attack of herpes zoster, and it is recognized that herpetic infections occur with greater frequency than normal in people suffering 0 from illness in which there is depression or incompetence of immunological reactions.

The myocardial and hepatic lymphocytic cellular infiltrates suggest involvement of the heart and liveror in the same way as the lungs and brain, thoughN more mildly. Further, it is possible that the orbital $\mathrm{W}_{\mathrm{S}}$ and periorbital tissue swelling which was present at 
one stage had a similar pathological basis, and it is interesting that clinical and histological features of a hypersensitivity reaction have previously been noted in orbital pseudotumours (Easton and Smith, 1961).

Both the clinical and the pathological aspects of this case of LIP are unusual. Histologically the pulmonary lesions were unequivocally those of lymphoid interstitial pneumonia. The affection of the brain by similar lesions, which was the immediate cause of death, and the milder simultaneous involvement of the heart and liver have not previously been described, and suggest that LIP may be a part of a more widely dispersed process. Our case offers clues suggesting that the disease process most likely resulted either from allergic hypersensitivity of cell-mediated type (type IV), or from invasion by a slow or latent virus.
REFERENCES

Easton, J. A., and Smith, W. T. (1961). Non-specific granuloma of orbit (Orbital Pseudotumour). J. Path. Bact., 82, 345-354.

Gell, P. G. H., and Coombs, R. R. A. (1968). Classification of allergic reactions responsible for clinical hypersensitivity and disease. In Clinical Aspects of Immunology, pp. 575-596. 2nd edn. Edited by P. G. H. Gell and R. R. A. Coombs. Blackwell: Oxford.

Liebow, A. A. (1968). New concepts and entities in pulmonary disease: lymphoid interstitial pneumonia (LIP). In The Lung, pp. 349-351. Edited by A. A. Liebow and D. E. Smith. Williams and Wilkins: Baltimore.

Seal, R. M. E., Hapke, E. J., Thomas, G. O., Meek, J. C., and Hayes, M. (1968). The pathology of the acute and chronic stages of farmer's lung. Thorax, 23, 469-489. 